\title{
Irisin: circulating levels in serum and its relation to gonadal axis
}

\author{
Yunyao Luo ${ }^{1}$ Xiaoyong Qiao ${ }^{2,3} \cdot$ Liangzhi Xu $\mathbb{C}^{2,3} \cdot$ Guoning Huang ${ }^{1}$
}

Received: 28 September 2021 / Accepted: 9 January 2022 / Published online: 17 January 2022

(c) The Author(s) 2022

\begin{abstract}
Irisin is an exercise-induced myokine/adipokine in mice and humans that plays an important role in 'browning' of white adipose tissue and has shown great potential as a treatment for some metabolic diseases, such as obesity, insulin resistance, and inflammation. The circulating irisin level is reported to be associated with exercise, obesity, diet, diseases, and exposure to different pharmacological agents. Several studies have attempted to characterize the role of irisin in PCOS and other reproductive diseases, but contradictory results have been reported. Our previous study showed that irisin may serve further functions in folliculogenesis and fertility. In this review, we present the current knowledge on the physiology of irisin and its role in gonadal axis. Firstly, we describe irisin circulating levels and speculate on the potential mechanisms involved in irisin secretion and regulation. Then, we focus on the irisin levels in PCOS, and explore the relationships between, BMI, insulin resistance, and hyperandrogenism. Finally, we present the results from animal interventional studies and in vitro experiments to investigate the relationship between irisin and gonadal axis, indicating its novel effects on reproduction and fertility.
\end{abstract}

Keywords Irisin $\cdot$ Polycystic ovary syndrome $\cdot$ HPG axis $\cdot$ Reproduction $\cdot$ Fertility

\section{Introduction}

Irisin is a novel cytokine that is mainly secreted from skeletal muscle and was discovered by Bostrom in 2012 [1]. It is cleaved from its precursor, fibronectin type III domain containing 5 (FNDC5), and secreted during exercise, adrenergic stimulation, or exposure to cold environments [2].

These authors contributed equally: Yunyao Luo, Xiaoyong Qiao

Liangzhi Xu

xuliangzhi_art@126.com

$\triangle$ Guoning Huang

gnhuang217@sina.com

1 Chongqing Key Laboratory of Human Embryo Engineering, Chongqing Reproduction and Genetics Institute, Chongqing Health Center for women and Children, No.64 Jin Tang Street, Yu Zhong District, Chongqing 400013, China

2 Reproductive Endocrinology and Regulation Laboratory West China Second University Hospital, Sichuan University, Chengdu, China

3 Department of Obstetrics and Gynecology, West China Second University Hospital, Sichuan University, Chengdu, People's Republic of China
Through binding to unknown receptors, irisin is proposed to induce 'browning' of white adipose tissue (WAT) [2, 3]. Studies have reported that irisin participates in energy expenditure, glucose uptake, and glycogenolysis by inhibiting gluconeogenesis, fat formation, and lipid accumulation and that it has potentially positive effects on glucose homeostasis and insulin sensitivity $[2,4,5]$. Therefore, based on these positive effects on metabolism, irisin is becoming a potential target for the treatment of metabolic diseases [6].

Reproductive health is closely related to the endocrine function of adipose tissue. Poor nutrition and low body mass are associated with the occurrence of infertility, whereas obesity and overweight also have adverse effects on fertility [7]. The impact of obesity on reproduction function, especially ovulatory disorders, are mainly attributable to endocrine mechanisms, which interfere with neuroendocrine and ovarian functions, and reduce the ovulation omeostatic. In men and women, body mass index (BMI) is reported to be negatively correlated with reproduction and fertility rate [8-10]. Obesity also has a negative impact on the outcomes of assisted reproductive technology (ART); the number of retrieved oocytes, ongoing pregnancy rate, and live birth rate are decreased in obesity [11-13]. Reproduction and fertility are closely linked to glucose and lipid metabolism. Hyperinsulinemia and insulin resistance 
(IR) interfere with follicle development and maturation, which can cause follicular dysplasia, ovulatory disorders, luteal phase defects, and poor fertility [14]. As irisin was discovered in components of the central nervous system (such as the brain and cerebrospinal fluid) and in reproductive organs (such as the seminiferous tubule, Leydig cells and epididymis) [15], its potential effects on reproduction have come into focus. Considering the positive effects of irisin on energy expenditure and glucose and lipid metabolism, irisin is likely to participate in reproductive health. Our previous study showed that irisin proteinknockout mice had disordered sex hormone metabolism and a significant decrease in fertility [16]. These findings not only showed the potential of irisin as a biochemical marker of many reproduction-related diseases but also led us to hypothesize that irisin might be directly involved in reproductive metabolism.

Some studies have focused on the effects of irisin on the secretion of sex hormones and the development of the gonads, but these studies have great heterogeneity, and some of the results need to be further verified. Therefore, the aim of this review is to summarize the currently existing evidence linking irisin with reproductive metabolism and to initially discuss the effects of irisin on reproduction and fertility.

\section{The secretion and regulation of irisin levels in different life phases}

In rodents and humans, several factors were found to affect the physiological levels of irisin in the body, including exercise, obesity, diet, diseases, and exposure to different pharmacological agents [17-21]. Whether there is a difference in irisin levels between sexes is still unknown. Data from some research showed that the content of irisin in girls was higher than that in boys [22] and higher in women than in men [23]; however, Zugel [24] and Scalzo [25] reported that irisin concentrations at rest showed no difference in between sexes in adults. At present, the reported circulating levels of irisin seem to differ greatly even in the same species, with reported concentrations in humans ranging from $0.01 \mathrm{ng} / \mathrm{ml}$ to $2000 \mathrm{ng} / \mathrm{ml}$ [26-30].

Many studies have investigated the effects of exercise on irisin secretion and have reported contradictory results. For example, some animal and human studies have shown an increase in circulating levels of irisin after exercise. Irisin levels were reported to be significantly higher in people who exercise regularly than in sedentary people [31-34]. However, a few studies have not found a clear positive or negative association between these factors [35-37]. The discrepancy in results can be explained by the type of exercises conducted in the studies; for instance, irisin concentrations were significantly increased in strenuous exercise studies but not in endurance training studies [38, 39]. Interestingly, Hernandaz et al. reported that the level of irisin in newborns who were delivered vaginally was significantly higher than that in newborns delivered via cesarean section [40]. Since the levels of irisin in mothers were comparable between the two groups, the result could be partly explained by exercise. During vaginal delivery, fetal adaptive rotation is accompanied by changes in body pressure, which is similar to the effect of exercise, resulting in increased irisin levels.

In newborns, the expression of FNDC5 is very low, as is the level of circulating irisin, most of which comes from the mother through the placental barrier [40, 41]. Wrann et al. reported that the expression of FNDC5 in the brains of mice increased gradually from the 0th day to the 20th day after birth [42]. Although that study was not focused on the change of irisin level in circulation, the increased expression of its precursor protein implied that the secretion pattern of irisin changed from 'maternal inheritance' to 'autonomous secretion' and then remained relatively stable. The secretion of irisin is variable in different life phases (such as puberty, the child-bearing period or menopause) and different stages (such as during pregnancy and around childbirth), which might be attributed to fluctuations in hormone levels. During the menstrual cycle, cyclical patterns of irisin levels could be observed, including that the circulating irisin in luteal phase increased by approximately $25 \%$ compared with that in follicular phase but gradually decreased along with the regression of the corpus luteum and returned to the baseline level in the early follicular phase [43]. In pubertal children and pregnant women, irisin levels increased significantly with the activation of the HPO axis [44], and irisin levels were positively correlated with gestational age during pregnancy [43]. The mechanism of the increase in irisin, which may be related to large changes in sex hormones (mainly estrogen and progesterone), is not clear. In women aged 41-82 years old, irisin levels decreased with age [18, 45-47], which might be explained by changes in estrogen levels. For example, a decrease in estrogen levels was accompanied by a significant decrease in irisin levels in menopausal women aged 40-56 years old. Certainly, the loss of muscle and bone mass may be one of the reasons for the decline in irisin in menopausal women. Interestingly, our co-workers have showed irisin has positive effects on bone metabolism [48, 49]. The decrease of irisin level in menopausal women may in turn aggravate the development of osteoporosis. These effects were consistent with our previous study showing irisin could ameliorate bone loss in ovariectomized mice [50].

To date, the mechanism of the dynamic changes in irisin levels remains unknown, and we speculate that the fluctuation of irisin may represent a self-protective metabolic 
mechanism. For instance, insulin resistance always increases physiologically during adolescence and pregnancy, which stimulates the secretion of irisin to maintain glucose and lipid homeostasis [18, 51]. In addition, a higher level of irisin can inhibit oxidative stress and inflammation, which is important to the maintenance of pregnancy [52-55].

\section{Irisin and PCOS}

Polycystic ovary syndrome (PCOS), a common endocrinopathy affecting 5-10\% of women of reproductive age [56]. The Rotterdam diagnostic criteria for PCOS are now internationally endorsed and are based on two of three features: oligo- or anovulation, hyperandrogenism (clinical or biochemical), and polycystic ovaries [56]. Insulin resistance (IR) and hyperinsulinemia play an important role in the pathophysiology and metabolic manifestations of PCOS, independent of but exacerbated by obesity [56, 57]. To date, data on circulating irisin levels between women with PCOS and controls have been derived from casecontrol studies, which have been inconclusive. Most studies have reported higher irisin levels in women with PCOS than in controls [58-61]. Ovarian drilling of polycystic ovaries results in a significant decrease in serum irisin levels [62]. However, some other studies reported similar [63, 64] or lower [65] circulating irisin levels in women with PCOS than in controls.

It has been reported that some patients with PCOS can resume ovulation after weight loss, indicating the adverse effects of obesity on PCOS [66]. Many studies have demonstrated a positive relationship between circulating irisin levels and BMI in healthy people as well as in PCOS patients [26, 67]. In PCOS patients, irisin concentrations were higher in overweight and obese women than that in women with normal weight $[59,63,68]$. Weight loss led to a significant decrease in circulating irisin (15\%), whereas weight regain returned irisin levels to baseline [59]. Therefore, the current controversial results of irisin level in PCOS may be related to the different baseline BMI level. The elevated irisin level may be a feedback mechanism to maintain metabolic balance in PCOS patients. Besides, it is also possible that there is irisin resistance in PCOS, similar to the insulin and leptin resistance observed in obesity and type 2 diabetes (T2DM) [43, 69, 70]. It should be highlighted that women with PCOS had a higher BMI than controls in most studies. In those patients, the increase of fat mass may induce the rising secretion of irisin, although these speculations remain to be elucidated [67].

Exercise and weight loss are well known to improve insulin sensitivity and prevent obesity and subsequent metabolic syndrome. As an exercise-induce cytokine, irisin, is reported to have multiple potential positive effects on glucose homeostasis and insulin sensitivity, such as promoting energy expenditure, glucose uptake, and glycogenolysis and reducing gluconeogenesis, adipogenesis, and lipid accumulation [2, 4, 5]. Although the etiology of PCOS remains unclear, IR plays an important role in its pathogenesis. Thus, the relationship between irisin and IR has become an interesting but controversial topic. Foda AA et al. [62] and Li et al. [59] indicated that circulating irisin was positively correlated with IR in PCOS women. After metformin treatment, the IR was improved in PCOS, and irisin level was reduced [59, 61]. On the other hand, Mareno et al. showed a negative relationship between irisin level and IR [18], whereas Choi [71] and Liu [19] reported no significant correlation between them.

In order to identify the role of irisin in IR, researchers focused on the relationship between irisin and insulin. In healthy people and patients with early diabetes, irisin has generally been negatively associated with insulin levels $[18,72]$. However, with the decrease in insulin sensitivity, this relationship becomes positive; for example, irisin levels have been reported to be higher in individuals with diabetes mellitus [73, 74]. Animal studies also have shown that irisin may promote insulin secretion [75]. Therefore, irisin and insulin level showed dynamic fluctuation during changes in glucose homeostasis; however, the causal relationship between them remains unclear. Future research should focus on the effect of irisin on insulin secretion and signaling and vice versa and on possible interactions between the two pathways that might affect glucose homeostasis.

In addition, researchers have discovered relationships between irisin and androgen levels [63, 68], bone metabolism [64], and metabolic syndrome [63] in women with PCOS and have observed some meaningful outcomes. Hyperandrogenism is an endocrinological disorder in women with PCOS. Some studies have reported there was a positive correction between irisin and androgen or free androgen index in PCOS women [68]. Because hyperandrogenism is an important inducer of IR, the relationship of irsin to hyperandrogenism and IR needs further investigation.

Altogether, the contradictory results related to irisin in PCOS may be attributed to the heterogeneity of the research. On the other hand, it may also embody that the body has experienced a decompensation period. The initial endocrine disorder in PCOS patients could stimulate more irisin secretion to maintain the counterbalance of metabolism. Along with the further aggravation of metabolic abnormalities that go far beyond the body's ability to compensate, a decrease in circulating irisin levels will occur. This is similar to the pattern of insulin levels in T2DM, which are elevated in the prediabetic state and reduced at an advanced stage of disease [59, 76]. 
In summary, the present review reported irisin may represent a novel PCOS biomarker and might directly contribute to the etiology of PCOS or indirectly affect the development of disordered metabolism (such as obesity, hyperandrogenism, impaired glucose tolerance, and insulin resistance) in PCOS [58]. The physiology and pathology of irisin in the development of PCOS remain to be further elucidated.

\section{Irisin and gonadal axis}

The incidence of menstrual disorders in the general female population is approximately 5\% [77], while it is as high as $12-79 \%$ of athletes [78-80]. Intense exercise is potentially harmful to the health of the female body, especially to reproductive function. Athletic menstrual cycle irregularities (AMI) are characterized by delayed menstruation, luteal phase defects, anovulation, hypomenorrhea and amenorrhea. The menstrual cycle can be gradually restored by reduced training intensity, and pregnancy can be achieved after stopping training. Fu et al. reported that the prolactin level in athletes with amenorrhea was significantly lower than that in athletes with normal menstrual cycles, while the levels of sex hormone binding globulin and growth hormone were significantly higher in the former group than in the latter, indicating that excessive exercise may interfere with the gonadal axis and influence hormone secretion, resulting in disordered reproductive function [81-83].

The hypothalamic-pituitary-ovarian (HPO) axis in females and the hypothalamic-pituitary-testicular (HPT) axis in males are the gonadal axes, which include hypothalamic neurons and their secreted gonadotropin-releasing hormone $(\mathrm{GnRH})$, pituitary gonadotropin cells, and their synthetic follicle stimulating hormone (FSH) and luteinizing hormone (LH), estrogen (mainly estradiol) and progesterone $(\mathrm{P})$ and testosterone $(\mathrm{T})$. They interact with each other to constitute a complete and coordinated network that plays a crucial role in the reproductive system [84, 85].

To elucidate the possible mechanism of AMI, researchers have proposed the "body composition hypothesis", "stress hypothesis" and "energy availability hypothesis" [86, 87]. At present, the "available energy hypothesis" proposed by Loucks et al. is generally accepted; this hypothesis holds that the imbalance between energy intake and energy expenditure is an important cause of menstrual disorders and that the inhibition of reproductive function is an adaptive response to reduced energy expenditure [88]. Many factors involved in regulating energy metabolism regulate reproductive health as well. For instance, insufficient energy intake reduced ghrelin production, which may inhibit reproductive functions by controlling $\mathrm{GnRH}, \mathrm{LH}$, and $\mathrm{E}_{2}$ release $[89,90]$. In addition, leptin and triiodothyronine have been reported to have positive effects on reproduction [91-95]. As mentioned above, there are significant changes in circulating irisin levels in puberty, pregnancy, and the postpartum period, when the functions of the HPG axis are active. However, the relationship between irisin and the HPG axis is still unknown. This section aims to summarize the effects of irisin on the development of the HPG axis and steroid hormone secretion to further explore the potential role of irisin in reproductive health.

\section{The relationship of irisin to $\mathrm{GnRH}$ and gonadotropin}

As important nerve centers, the hypothalamus and pituitary regulate downstream organs and hormone production through GnRH and gonadotropin, respectively. FNDC5 is mainly expressed in the proximal pars distalis (PPD) of the pituitary [96]. The existing data showed that irisin has positive effects on pituitary functions. In vivo, irisin could promote the secretion of FSH and LH in female rats [97, 98]. The female mice lacking irisin, showed lower concentrations of FSH and LH than those in wild-type mice [16]. Besides, in vitro, irisin treatment increased the expression of FSH and $\mathrm{LH}$ in the pituitary cells by improving the stability of transcription [96]. These results indicate that irisin may play a GnRH-like role in the HPG axis.

However, irisin showed opposing effects on gonadotropins when in combination with GnRH. For example, Ulker et al. showed that irisin exposure reduced the expression of GnRH in the hypothalamus, and delayed the onset of puberty (including significantly delayed vaginal opening time and estrous cycle) in female rats [98]. Besides, in vitro experiments, GnRH could promote the secretion of FSH and LH by pituitary cells when administered singlely, while the stimulating effects could be inhibited by irisin treatment in combination [96, 99]. According to the present results, we assumed that there may be two reasons for the negative effects of irisin on GnRH. First, irisin could promote the secretion of FSH and $\mathrm{LH}$, inhibiting the release of GnRH through negative feedback. Second, we speculate that the effect of irisin is similar to that of gonadotropin-releasing hormone agonist (GnRH-a). The specific receptor of irisin in pituitary gonadotropin-secreting cells has thus far not been found. Therefore, irisin may bind to GnRH receptors on the cell surface, followed by a decrease in the number of available receptors and an increase in receptor internalization, resulting in pituitary desensitization, which inhibits the release of gonadotropins. The mechanism is similar to the process of pituitary suppression used in assisted reproductive technology $[100,101]$. 
Therefore, irisin could promote the expression of FSH and $\mathrm{LH}$, and on the one hand, irisin could compete with GnRH to inhibit the secretion of FSH and LH. These dual effects of irisin occur at the same time and interact with each other, and changes in circulating hormone levels are achieved when one activity is dominant.

\section{Irisin and ovarian/testicular and sex hormones}

The ovary and testis, as downstream organs, are directly regulated by gonadotropin. The present data suggest that the interaction of irisin with FSH and LH may affect the development of the sexual gland. For example, irisin can promote the development of convoluted seminiferous tubules and the motility of germ cells in male rats [98]. FNDC5, the precursor protein of irisin, can promote the development of mouse ovaries, as reported by Bastu [97] et al., showing a significant increase in the number of primary and secondary follicles. Consistent with their results, our previous study showed that FNDC5-knockout mice had a decreased number of antral follicles. In contrast, some studies have reported that irisin has negative effects on ovaries and testes, significantly reducing the number of vegetative cells and Leydig cells, sperm density and mobility in male rats under irisin exposure [102], and causing a decrease in the number of primary follicles and a significant increase in ovarian fibrosis in female mice [98]. It has been reported that the physiological effect of irisin is concentration-dependent, which can partly explain the different or even opposite effects of irisin in metabolism. Irisin can promote the expression of LH in the pituitary gland of tilapia at a concentration of $1 \mathrm{nM}$, while the lowest concentration that promotes the expression of FSH is $10 \mathrm{nM}$ [96]. In porcine ovarian granulosa cells, irisin at a low concentration $(50 \mathrm{ng} / \mathrm{ml})$ could promote progesterone secretion, while a high concentration $(150 \mathrm{ng} / \mathrm{ml})$ could inhibit the production of progesterone [103]. In addition, the effect of irisin is time-dependent. Wagner [104] et al. reported that long-term ( $>7$ days) treatment with a supraphysiological dose (100-1000 ng/ml) of irisin could inhibit the expression of follicle stimulating hormone receptor (FSHR), while a physiological dose (10-100 ng/ml) of irisin or short-term $(48 \mathrm{~h})$ treatment had no significant effect on the expression of FSHR. Certainly, the use of different species and experimental protocols lead to high heterogeneity as well.

In terms of reproduction, nitric oxide (NO) can promote the meiosis of oocytes and the development of follicles and shows dual effects on follicular atresia [105, 106]. Interestingly, recent studies have reported the relationship between irisin and NO in the ovary. Bastu et al. reported that treatment with FNDC5 can promote the expression of nitric oxide synthase (NOS) in mouse ovaries [97] while significantly decreasing the expression of NO under irisin exposure in porcine ovarian granulosa cells [103]. The possible reason for the discrepancy is that NO can both promote and inhibit apoptosis. Moreover, the expression of $\mathrm{NO}$ is also affected by the complex environment in vivo; the atresia of follicles is regulated by many pathways, and the secretion of NO is affected by many factors. In short, there is a lack of evidence related to the direct effect of irisin on gonadal development and germ cell functions.

Sex steroids, including androgens, estrogens, and progesterone, are known to have widespread physiological actions in the reproductive system [107]. The present data showed a positive relationship between irisin and $\mathrm{E}_{2}$ levels [46, 98, 99], which may be related to the effect of irisin on FSH and LH. In addition, the effect of irisin on $\mathrm{E}_{2}$ synthesis could be due to irisin affecting ovarian aromatase activity. Our previous studies have confirmed that irisin can directly promote estrogen secretion by promoting the expression of CYP19A1, which is the rate-limiting enzyme of estrogen synthesis [16, 108]. To date, limited studies have described the relationship between irisin and progesterone. Our study initially reported an elevated progesterone level in FNDC5knockout mice, which may be due to the downregulation of Akr1c18 gene expression, which in turn is mainly related to the removal of progesterone from the body. The results of irisin and androgen studies are inconsistent. Some studies observed a positive correlation between circulating irisin and testosterone levels [102, 109], while others showed a negative relationship between irisin and testosterone levels [59, 68] or no significant correlation [46].

\section{The other effects of irisin}

Abnormal reproductive function eventually leads to decreased fertility and even infertility. The present data suggest that the infertility rate in China is between $12 \%$ and $15 \%$; that is, more than 50 million couples are infertile, and infertility has become a common and serious public health problem [110, 111]. Reproduction and fertility are closely related to energy metabolism and the endocrine function of adipose tissue. Obesity or insufficient fat content will adversely affect fertility [112]. As regulators of energy expenditure, leptin and insulin contribute to reproductive health as well. For example, leptin participates in the regulation of energy, and its deficiency reduces endometrial receptivity, while insulin is a regulator of glucose and lipid metabolism, as well as endometrial receptivity [113, 114]. In addition, endometrial receptivity is significantly decreased in obesity [115]. Li et al. demonstrated that irisin may improve the receptivity of the endometrium by 
promoting the expression of leukemia inhibitory factor and integrin $\alpha v \beta 3$ [116]. In addition, reproductive function is regulated by many other metabolic factors, such as the growth hormone axis (GH/IGFs), which is very important in gonadal development and fertility, including promoting oocyte maturation, granulosa cell proliferation, differentiation, and steroid hormone synthesis [117, 118]. Energy metabolism directly affects the secretion of GH and IGF-I, and some studies have reported that the levels of FNDC5 and/or irisin in circulation are positively correlated with the activity of the GH/IGF-I axis [46, 119, 120]. Therefore, irisin may have an indirect effect on fertility through the action of the growth hormone axis. Our previous study initially confirmed the effect of irisin on fertility in mice. In that study, irisin-deficient mice showed poor fertility, which was mainly characterized by significant decreases in the birth rate, average litter size per cage, the survival rate of newborn mice, and a significantly prolonged average time to litter production [76]. Due to the lack of available evidence, the specific effects and mechanisms of irisin on fertility are unclear, and more in-depth research is required to develop the field.

\section{Conclusion}

Irisin is a novel myokine/adipokine that is implicated in the regulation of a variety of endocrine and metabolic functions, such as promoting WAT browning and glucose homeostasis. Irisin is appealing, with the potential to bridge our knowledge gaps related to the connection between exercise and beneficial effects on metabolic diseases. The circulating irisin level is likely related to sex, age, and physiological status. Clinical studies have indicated an association between circulating levels of irisin and PCOS. Interactions between irisin, BMI, insulin, androgens, or other hormones that are involved in the endocrine metabolic abnormalities in PCOS should be researched in the future. Many studies have underscored the positive role of irisin in reproductive health. Through its functions in the HPG axis, irisin contributes to the development of the ovary and testis and the secretion of steroids. Therefore, further research is required to confirm the relationship between irisin and other myokines and their causal relations. One crucial further step is to identify the specific receptor of irisin in the ovary and testis to characterize its mode of action, laying a solid foundation for the development of drugs for the treatment of various metabolic disorders and declining fertility.

Author contributions Y.L. prepared the original draft of the manuscript; L.X. and G.H. reviewed and edited the manuscript; Y.L. and X.Q. wrote and reviewed the manuscript.
Funding This review was supported by Research Projects of The National Natural Science Fund (81971354) and The National Natural Science Fund (81701413).

\section{Compliance with ethical standards}

Conflict of interest The authors declare no competing interests.

Publisher's note Springer Nature remains neutral with regard to jurisdictional claims in published maps and institutional affiliations.

Open Access This article is licensed under a Creative Commons Attribution 4.0 International License, which permits use, sharing, adaptation, distribution and reproduction in any medium or format, as long as you give appropriate credit to the original author(s) and the source, provide a link to the Creative Commons license, and indicate if changes were made. The images or other third party material in this article are included in the article's Creative Commons license, unless indicated otherwise in a credit line to the material. If material is not included in the article's Creative Commons license and your intended use is not permitted by statutory regulation or exceeds the permitted use, you will need to obtain permission directly from the copyright holder. To view a copy of this license, visit http://creativecommons. org/licenses/by/4.0/.

\section{References}

1. P. Boström et al. A PGC1- $\alpha$-dependent myokine that drives brown-fat-like development of white fat and thermogenesis. Nature 481(7382), 463-468 (2012).

2. N. Perakakis et al. Physiology and role of irisin in glucose homeostasis. Nat. Rev. Endocrinol. 13(6), 324-337 (2017).

3. F. Villarroya, Irisin, turning up the heat. Cell Metab. 15(3), 277-278 (2012).

4. T.Y. Liu et al. Irisin inhibits hepatic gluconeogenesis and increases glycogen synthesis via the PI3K/Akt pathway in type 2 diabetic mice and hepatocytes. Clin. Sci. (Lond.) 129(10), 839-850 (2015).

5. L. Mo et al. Irisin is regulated by CAR in liver and is a mediator of hepatic glucose and lipid metabolism. Mol. Endocrinol. 30(5), 533-542 (2016).

6. S.A. Polyzos et al. Irisin in metabolic diseases. Endocrine 59(2), 260-274 (2018).

7. D.R. Meldrum, Introduction: obesity and reproduction. Fertil. Steril. 107(4), 831-832 (2017).

8. S.L. Loy et al. Female adiposity and time-to-pregnancy: a multiethnic prospective cohort. Hum. Reprod. 33(11), 2141-2149 (2018).

9. M.B. Cavalcante et al. Obesity and recurrent miscarriage: a systematic review and meta-analysis. J. Obstet. Gynaecol. Res 45 (1), 30-38 (2019).

10. A. Talmor, B. Dunphy, Female obesity and infertility. Best. Pr. Res Clin. Obstet. Gynaecol. 29(4), 498-506 (2015).

11. R. Mushtaq et al. Effect of male body mass index on assisted reproduction treatment outcome: an updated systematic review and meta-analysis. Reprod. Biomed. Online 36(4), 459-471 (2018).

12. A. Setti et al. Association between parental anthropometric measures and the outcomes of intracytoplasmic sperm injection cycles. J. Assist Reprod. Genet 36(3), 461-471 (2019).

13. P.R. Supramaniam et al. The correlation between raised body mass index and assisted reproductive treatment outcomes: a 
systematic review and meta-analysis of the evidence. Reprod. Health 15(1), 34 (2018).

14. E. Silvestris et al. Obesity as disruptor of the female fertility. Reprod. Biol. Endocrinol. 16(1), 22 (2018).

15. S. Aydin et al. A comprehensive immunohistochemical examination of the distribution of the fat-burning protein irisin in biological tissues. Peptides 61, 130-136 (2014).

16. Y. Luo et al. Irisin deletion induces a decrease in growth and fertility in mice. Reprod. Biol. Endocrinol. 19(1), 22 (2021).

17. B.M. Varela-Rodríguez et al. FNDC5 expression and circulating irisin levels are modified by diet and hormonal conditions in hypothalamus, adipose tissue and muscle. Sci. Rep. 6, 29898 (2016).

18. J.M. Moreno-Navarrete et al. Irisin is expressed and produced by human muscle and adipose tissue in association with obesity and insulin resistance. J. Clin. Endocrinol. Metab. 98(4), E769-E778 (2013).

19. J.J. Liu et al. Lower circulating irisin is associated with type 2 diabetes mellitus. J. Diabetes Complications 27(4), 365-369 (2013).

20. A. Zybek-Kocik et al. Time-dependent irisin concentration changes in patients affected by overt hypothyroidism. Endokrynol. Pol. 67(5), 476-480 (2016).

21. M.P. Jedrychowski et al. Detection and quantitation of circulating human irisin by tandem mass spectrometry. Cell Metab. 22 (4), 734-740 (2015).

22. N.M. Al-Daghri et al. Irisin as a predictor of glucose metabolism in children: sexually dimorphic effects. Eur. J. Clin. Invest 44(2), 119-124 (2014).

23. A.D. Anastasilakis, et al. Circulating irisin in healthy, young individuals: day-night rhythm, effects of food intake and exercise, and associations with gender, physical activity, diet, and body composition. J. Clin. Endocrinol. Metab. 99(9), 3247-3255 (2014).

24. M. Zügel et al. The role of sex, adiposity, and gonadectomy in the regulation of irisin secretion. Endocrine 54(1), 101-110 (2016).

25. R.L. Scalzo et al. Regulators of human white adipose browning: evidence for sympathetic control and sexual dimorphic responses to sprint interval training. PLoS One 9(6), e90696 (2014).

26. A.B. Crujeiras, M. Pardo, F.F. Casanueva, Irisin: 'fat' or artefact. Clin. Endocrinol. (Oxf.) 82(4), 467-474 (2015).

27. U. Elbelt, T. Hofmann, A. Stengel, Irisin: what promise does it hold? Curr. Opin. Clin. Nutr. Metab. Care 16(5), 541-547 (2013).

28. T. Hofmann, U. Elbelt, A. Stengel, Irisin as a muscle-derived hormone stimulating thermogenesis-a critical update. Peptides 54, 89-100 (2014).

29. S.A. Polyzos, C.S. Mantzoros, An update on the validity of irisin assays and the link between irisin and hepatic metabolism. Metabolism 64(9), 937-942 (2015).

30. S.A. Polyzos, H. Mathew, C.S. Mantzoros, Irisin: a true, circulating hormone. Metabolism 64(12), 1611-1618 (2015).

31. E. Miyamoto-Mikami et al. Endurance training-induced increase in circulating irisin levels is associated with reduction of abdominal visceral fat in middle-aged and older adults. PLoS One 10(3), e0120354 (2015).

32. X.Q. Yang et al. Swimming intervention mitigates HFD-induced obesity of rats through PGC- $1 \alpha$-irisin pathway. Eur. Rev. Med Pharm. Sci. 20(10), 2123-2130 (2016).

33. Y. Tsuchiya et al. High-intensity exercise causes greater irisin response compared with low-intensity exercise under similar energy consumption. Tohoku J. Exp. Med 233(2), 135-140 (2014).

34. Y. Tsuchiya et al. Resistance exercise induces a greater irisin response than endurance exercise. Metabolism 64(9), 1042-1050 (2015).
35. S. Raschke et al. Evidence against a beneficial effect of irisin in humans. PLoS One 8(9), e73680 (2013).

36. T. Kurdiova et al. Exercise-mimicking treatment fails to increase Fndc5 mRNA \& irisin secretion in primary human myotubes. Peptides 56, 1-7 (2014).

37. A. Besse-Patin et al. Effect of endurance training on skeletal muscle myokine expression in obese men: identification of apelin as a novel myokine. Int J. Obes. (Lond.) 38(5), 707-713 (2014).

38. F. Norheim, et al. The effects of acute and chronic exercise on PGC- $1 \alpha$, irisin and browning of subcutaneous adipose tissue in humans. Febs j. 281(3), 739-749 (2014).

39. J. Brenmoehl et al. Irisin is elevated in skeletal muscle and serum of mice immediately after acute exercise. Int J. Biol. Sci. 10(3), 338-349 (2014).

40. M. Hernandez-Trejo et al. Relationship between irisin concentration and serum cytokines in mother and newborn. PLoS One 11(11), e0165229 (2016).

41. N.M. Al-Daghri et al. Maternal inheritance of circulating irisin in humans. Clin. Sci. (Lond.) 126(12), 837-844 (2014).

42. C.D. Wrann et al. Exercise induces hippocampal BDNF through a PGC-1 $\alpha /$ FNDC5 pathway. Cell Metab. 18(5), 649-659 (2013).

43. M.F. Garcés et al. Irisin levels during pregnancy and changes associated with the development of preeclampsia. J. Clin. Endocrinol. Metab. 99(6), 2113-2119 (2014).

44. T. Reinehr et al. Irisin and its relation to insulin resistance and puberty in obese children: a longitudinal analysis. J. Clin. Endocrinol. Metab. 100(5), 2123-2130 (2015)

45. A.D. Anastasilakis et al. Circulating irisin is associated with osteoporotic fractures in postmenopausal women with low bone mass but is not affected by either teriparatide or denosumab treatment for 3 months. Osteoporos. Int 25(5), 1633-1642 (2014).

46. J.Y. Huh et al. FNDC5 and irisin in humans: I. Predictors of circulating concentrations in serum and plasma and II. mRNA expression and circulating concentrations in response to weight loss and exercise. Metabolism 61(12), 1725-1738 (2012).

47. T. Ebert et al. Serum levels of the myokine irisin in relation to metabolic and renal function. Eur. J. Endocrinol. 170(4), 501-506 (2014).

48. Y. Ma et al. Irisin promotes proliferation but inhibits differentiation in osteoclast precursor cells. Faseb j. 32(11), 5813-5823 (2018).

49. X. Qiao et al. Irisin promotes osteoblast proliferation and differentiation via activating the MAP kinase signaling pathways. Sci. Rep. 6, 18732 (2016).

50. Y. Luo et al. Irisin ameliorates bone loss in ovariectomized mice. Climacteric 23(5), 496-504 (2020).

51. J.Y. Huh et al. Exercise-induced irisin secretion is independent of age or fitness level and increased irisin may directly modulate muscle metabolism through AMPK activation. J. Clin. Endocrinol. Metab. 99(11), E2154-E2161 (2014).

52. M.J. Park et al. New role of irisin in hepatocytes: the protective effect of hepatic steatosis in vitro. Cell Signal 27(9), 1831-1839 (2015).

53. S. Hauguel-de Mouzon, M. Guerre-Millo, The placenta cytokine network and inflammatory signals. Placenta 27(8), 794-798 (2006).

54. G. Mor et al. Inflammation and pregnancy: the role of the immune system at the implantation site. Ann. N. Y Acad. Sci. 1221(1), 80-87 (2011).

55. J. Dong et al. Inhibition of myostatin in mice improves insulin sensitivity via irisin-mediated cross talk between muscle and adipose tissues. Int J. Obes. (Lond.) 40(3), 434-442 (2016).

56. Sood, M., et al. Sood M, Zweig S B, Tolentino M C, et al. Polycystic Ovary Syndrome[M]. Springer International Publishing, 2017. 
57. R.K. Meier, Polycystic ovary syndrome. Nurs. Clin. North Am. 53(3), 407-420 (2018).

58. C.L. Chang et al. Circulating irisin and glucose-dependent insulinotropic peptide are associated with the development of polycystic ovary syndrome. J. Clin. Endocrinol. Metab. 99(12), E2539-E2548 (2014).

59. M. Li et al. Elevated circulating levels of irisin and the effect of metformin treatment in women with polycystic ovary syndrome. J. Clin. Endocrinol. Metab. 100(4), 1485-1493 (2015).

60. M.S. Bostanc1 et al. Serum irisin levels in patients with polycystic ovary syndrome. Eur. Rev. Med Pharm. Sci. 19(23), 4462-4468 (2015).

61. A. Adamska et al. Serum irisin and its regulation by hyperinsulinemia in women with polycystic ovary syndrome. Endocr. J. 63(12), 1107-1112 (2016).

62. A.A. Foda, E.A. Foda, Z.H. El-Said, Serum irisin levels in polycystic ovary syndrome after ovarian drilling. Diabetes Metab. Syndr. 13(2), 1463-1468 (2019).

63. K. Pukajło et al. Irisin plasma concentration in PCOS and healthy subjects is related to body fat content and android fat distribution. Gynecol. Endocrinol. 31(11), 907-911 (2015).

64. S. Gao et al. The relationships of irisin with bone mineral density and body composition in PCOS patients. Diabetes Metab. Res Rev. 32(4), 421-428 (2016).

65. R. Abali et al. Implications of circulating irisin and Fabp4 levels in patients with polycystic ovary syndrome. J. Obstet. Gynaecol. 36(7), 897-901 (2016).

66. R. Pasquali, A. Gambineri, U. Pagotto, The impact of obesity on reproduction in women with polycystic ovary syndrome. Bjog 113(10), 1148-1159 (2006).

67. A.B. Crujeiras et al. Association between circulating irisin levels and the promotion of insulin resistance during the weight maintenance period after a dietary weight-lowering program in obese patients. Metabolism 63(4), 520-531 (2014).

68. H. Li et al. Free androgen index and Irisin in polycystic ovary syndrome. J. Endocrinol. Invest 39(5), 549-556 (2016).

69. G. Sesti et al. High circulating irisin levels are associated with insulin resistance and vascular atherosclerosis in a cohort of nondiabetic adult subjects. Acta Diabetol. 51(5), 705-713 (2014).

70. R.C. Frederich et al. Leptin levels reflect body lipid content in mice: evidence for diet-induced resistance to leptin action. Nat. Med 1(12), 1311-1314 (1995).

71. Y.K. Choi et al. Serum irisin levels in new-onset type 2 diabetes. Diabetes Res Clin. Pr. 100(1), 96-101 (2013).

72. H. Staiger et al. Common genetic variation in the human FNDC5 locus, encoding the novel muscle-derived 'browning' factor irisin, determines insulin sensitivity. PLoS One 8(4), e61903 (2013).

73. I. Ates et al. Factors associated with increased irisin levels in the type 1 diabetes mellitus. Endocr. Regul. 51(1), 1-7 (2017).

74. D. Espes, J. Lau, P.O. Carlsson, Increased levels of irisin in people with long-standing Type 1 diabetes. Diabet. Med 32(9), 1172-1176 (2015).

75. Y. Zhang et al. Irisin stimulates browning of white adipocytes through mitogen-activated protein kinase p38 MAP kinase and ERK MAP kinase signaling. Diabetes 63(2), 514-525 (2014).

76. K.H. Park et al. Circulating irisin in relation to insulin resistance and the metabolic syndrome. J. Clin. Endocrinol. Metab. 98(12), 4899-4907 (2013).

77. W.L. Xu et al. Exercise and female reproductive endocrine. Sports Sci. 04, 28-29 (1993).

78. A.Z. Hoch et al. Prevalence of the female athlete triad in high school athletes and sedentary students. Clin. J. Sport Med 19(5), 421-428 (2009).
79. J.M. Thein-Nissenbaum et al. Menstrual irregularity and musculoskeletal injury in female high school athletes. J. Athl. Train. 47(1), 74-82 (2012).

80. J. Thein-Nissenbaum, E. Hammer, Treatment strategies for the female athlete triad in the adolescent athlete: current perspectives. Open Access J. Sports Med 8, 85-95 (2017).

81. C. Enea et al. Circulating androgens in women: exercise-induced changes. Sports Med 41(1), 1-15 (2011).

82. A. Leal-Cerro et al. Mechanisms underlying the neuroendocrine response to physical exercise. J. Endocrinol. Invest 26(9), 879-885 (2003).

83. J. Witkoś, P. Wróbel, Menstrual disorders in amateur dancers. BMC Women's Health 19(1), 87 (2019).

84. A. Acevedo-Rodriguez et al. Emerging insights into hypothalamic-pituitary-gonadal axis regulation and interaction with stress signalling. J. Neuroendocrinol. 30(10), e12590 (2018).

85. Y. Tobari et al. A new pathway mediating social effects on the endocrine system: female presence acting via norepinephrine release stimulates gonadotropin-inhibitory hormone in the paraventricular nucleus and suppresses luteinizing hormone in quail. J. Neurosci. 34(29), 9803-9811 (2014).

86. R.E. Frisch, J.W. McArthur, Menstrual cycles: fatness as a determinant of minimum weight for height necessary for their maintenance or onset. Science 185(4155), 949-951 (1974)

87. M. van Bodegom, J.R. Homberg, M. Henckens, Modulation of the hypothalamic-pituitary-adrenal axis by early life stress exposure. Front Cell Neurosci. 11, 87 (2017).

88. A.B. Loucks, M. Verdun, E.M. Heath, Low energy availability, not stress of exercise, alters LH pulsatility in exercising women. J. Appl Physiol. (1985) 84(1), 37-46 (1998).

89. N.I. Williams et al. Estrogen and progesterone exposure is reduced in response to energy deficiency in women aged 25-40 years. Hum. Reprod. 25(9), 2328-2339 (2010).

90. R. Fernandez-Fernandez et al. Novel signals for the integration of energy balance and reproduction. Mol. Cell Endocrinol. 254255, 127-132 (2006).

91. C.R. Barb, G.J. Hausman, C.A. Lents, Energy metabolism and leptin: effects on neuroendocrine regulation of reproduction in the gilt and sow. Reprod. Domest. Anim. 43 Suppl 2, 324-330 (2008).

92. E. Gregoraszczuk et al. Action of IGF-I on expression of the long form of the leptin receptor $(\mathrm{ObRb})$ in the prepubertal period and throughout the estrous cycle in the mature pig ovary. J. Reprod. Dev. 53(2), 289-295 (2007).

93. E.L. Gregoraszczuk et al. Gh and IGF-I increase leptin receptor expression in prepubertal pig ovaries: the role of leptin in steroid secretion and cell apoptosis. Acta Vet. Hung. 54(3), 413-426 (2006).

94. N.I. Williams et al. Evidence for a causal role of low energy availability in the induction of menstrual cycle disturbances during strenuous exercise training. J. Clin. Endocrinol. Metab. 86 (11), 5184-5193 (2001).

95. A. Tropea et al. Ghrelin affects the release of luteolytic and luteotropic factors in human luteal cells. J. Clin. Endocrinol. Metab. 92(8), 3239-3245 (2007).

96. Q. Jiang et al. Irisin stimulates gonadotropins gene expression in tilapia (Oreochromis niloticus) pituitary cells. Anim. Reprod. Sci. 185, 140-147 (2017).

97. E. Bastu et al. Effects of irisin and exercise on metabolic parameters and reproductive hormone levels in high-fat diet-induced obese female mice. Reprod. Sci. 25(2), 281-291 (2018).

98. N. Ulker et al. Irisin may have a role in pubertal development and regulation of reproductive function in rats. Reproduction $\mathbf{1 6 0}$ (2), 281-292 (2020) 
99. L. Poretsky et al. Reproductive effects of irisin: Initial in vitro studies. Reprod. Biol. 17(3), 285-288 (2017).

100. P. Humaidan et al. Ovarian response and pregnancy outcome related to mid-follicular $\mathrm{LH}$ levels in women undergoing assisted reproduction with GnRH agonist down-regulation and recombinant FSH stimulation. Hum. Reprod. 17(8), 2016-2021 (2002).

101. J. Ren et al. Does prolonged pituitary down-regulation with gonadotropin-releasing hormone agonist improve the live-birth rate in in vitro fertilization treatment?. Fertil. Steril. 102(1), 75-81 (2014).

102. S. Tekin et al. Effects of intracerebroventricular administration of irisin on the hypothalamus-pituitary-gonadal axis in male rats. J. Cell Physiol. 234(6), 8815-8824 (2019).

103. G. Basini et al. The myokine irisin: localization and effects in swine late medium and large antral ovarian follicle. Domest. Anim. Endocrinol. 74, 106576 (2021).

104. I.V. Wagner et al. Adipocytokines may delay pubertal maturation of human Sertoli cells. Reprod Fertil Dev, 31(8), 1395-1400 (2019).

105. A. Jablonka-Shariff, L.M. Olson, Nitric oxide is essential for optimal meiotic maturation of murine cumulus-oocyte complexes in vitro. Mol. Reprod. Dev. 55(4), 412-421 (2000).

106. Q. Chen et al. Cross-Talk between Fas/Fas ligand system and nitric oxide in the pathway subserving granulosa cell apoptosis: a possible regulatory mechanism for ovarian follicle atresia. Endocrinology 146(2), 808-815 (2005).

107. R. Lanes, Sex hormone priming. J. Pediatr. Endocrinol. Metab. 24(1-2), 7-8 (2011).

108. N. Naderpoor et al. Obesity and polycystic ovary syndrome. Minerva Endocrinol. 40(1), 37-51 (2015).

109. Z. Kamenov et al. Irisin and testosterone in men with metabolic syndrome. Horm. Metab. Res 49(10), 755-759 (2017).
110. H.F. Huang et al. Analysis on the current situation and trend of infertility. Chin. J. Practical Gynecol. Obstet. 29(09), 688-690 (2013).

111. X.Y. Zheng et al. Inter provincial differences of infertility among first married women in China and its relationship with economic development. Chin. J. Public Health 28(11), 1411-1413 (2012).

112. R. Pasquali, L. Patton, A. Gambineri, Obesity and infertility. Curr. Opin. Endocrinol. Diabetes Obes. 14(6), 482-487 (2007).

113. Y.J. Yang et al. Leptin-directed embryo implantation: leptin regulates adhesion and outgrowth of mouse blastocysts and receptivity of endometrial epithelial cells. Anim. Reprod. Sci. 92 (1-2), 155-167 (2006).

114. $\mathrm{R}$. Li et al. Mice endometrium receptivity in early pregnancy is impaired by maternal hyperinsulinemia. Mol. Med Rep. 15(5), 2503-2510 (2017).

115. M. Kasum et al. The role of female obesity on in vitro fertilization outcomes. Gynecol. Endocrinol. 34(3), 184-188 (2018).

116. C. Li et al. Effect of irisin on endometrial receptivity of rats with polycystic ovary syndrome. Gynecol. Endocrinol. 35(5), 395-400 (2019).

117. E. Kiapekou et al. Effects of GH and IGF-I on the in vitro maturation of mouse oocytes. Hormones (Athens) 4(3), 155-160 (2005).

118. S. Mazerbourg et al. The insulin-like growth factor system: a key determinant role in the growth and selection of ovarian follicles? a comparative species study. Reprod. Domest. Anim. 38(4), 247-258 (2003).

119. S. Srinivasa et al. FNDC5 relates to skeletal muscle IGF-I and mitochondrial function and gene expression in obese men with reduced growth hormone. Growth Horm. IGF Res 26, 36-41 (2016).

120. J.Y. Huh et al. Irisin stimulates muscle growth-related genes and regulates adipocyte differentiation and metabolism in humans. Int J. Obes. (Lond.) 38(12), 1538-1544 (2014). 